\title{
Bioequivalence of the Rilpivirine/Emtricitabine/Tenofovir Alafenamide Single-Tablet Regimen
}

Zack J, Chuck S*, Chu H, Graham H, Cao H, Tijerina M, West S, Fang L, Quirk E and Kearney B

Gilead Sciences Inc., Foster City, CA, United States

\begin{abstract}
Rilpivirine/emtricitabine/tenofovir alafenamide (RPV/FTC/TAF) is the next advancement in once-daily antiretroviral single-tablet regimen (STR) for the treatment of HIV-1 in adults. Tenofovir disoproxil fumarate (TDF), an oral prodrug of tenofovir (TFV), is a preferred nucleotide reverse transcriptase inhibitor, but it is also associated with nephrotoxicity and reduced bone mineral density (BMD). TDF has been replaced by tenofovir alafenamide (TAF), a novel, oral prodrug of TFV with a different metabolic pathway leading to $91 \%$ lower circulating plasma concentrations of TFV. Differences with TAF have resulted in less nephrotoxicity and less reduction or increase in BMD in clinical trials. This study evaluated the pharmacokinetics and bioequivalence of the components of RPV/FTC/TAF (25/200/25 mg) STR to the references of RPV $(25 \mathrm{mg})$ single tablet and elvitegravir (E)/cobicistat (C)/FTC (F)/TAF $(150 / 150 / 200 / 10 \mathrm{mg})$ in healthy subjects. Ninety-six subjects were randomized in a single-dose, open-label, 3-way, 6-sequence, crossover Phase 1 study; two subjects did not complete the study. Serial blood samples were obtained over 336 hours for RPV/FTC/ TAF and RPV and over 144 hours for E/C/F/TAF following oral administration of each treatment, and pharmacokinetic parameters were calculated. Formulation bioequivalence was assessed by $90 \%$ confidence intervals (Cls) for the geometric least-squares mean (GLSM) ratios of pharmacokinetic parameter AUC AUC and C for each component of RPV/FTC/TAF compared to RPV and $\mathrm{E} / \mathrm{C} / \mathrm{F} / \mathrm{TAF}$. The test and reference treatments administered under fed conditions were generally well tolerated. The $90 \% \mathrm{Cls}$ for the GLSM ratios of the primary pharmacokinetic parameter $A \cup C_{\text {last }}, A \cup C_{\text {inf }}$, and $C_{\max }$ for test versus reference treatments were within the protocol-specified bioequivalence boundary of $80 \%$ to $125 \%$ for FTC, RPV, and TAF. RPV/FTC/TAF is the next advancement in once-daily STR for the treatment of HIV-1 in adults. Once approved, it will add to the armamentarium of STRs available for efficacious and safe therapies.
\end{abstract}

Keywords: Bioequivalence; Rilpivirine; Tenofovir alafenamide; Emtricitabine; HIV-1; Pharmacokinetics; Single-tablet regimen

\section{Introduction}

The Department of Health and Human Services (DHHS) guidelines for use of antiretroviral agents in HIV-1-infected adults and adolescents has designated the combination of emtricitabine (FTC) and tenofovir disoproxil fumarate (TDF) as a preferred nucleoside/ nucleotide reverse transcriptase inhibitor (NRTI/NtRTI) backbone [1]. However, safety concerns with TDF, an oral prodrug of tenofovir (TFV), include nephrotoxicity and reduced bone mineral density (BMD) [2]. Advances in antiretroviral therapy (ART) have resulted in increased life expectancies for HIV-infected patients and a refocused attention on the safety profile of ART as patients age given the increased prevalence of comorbidities at a younger age than their HIV-negative counterparts [3]. Tenofovir alafenamide (TAF), a novel, oral prodrug of TFV, has a different metabolic pathway from TDF that enhances lymphocyte delivery of TFV, resulting in $91 \%$ lower circulating plasma concentrations of TFV while maintaining similar intracellular levels of the active phosphorylated metabolite TFV-diphosphate (TFV-DP) [4]. These differences seen with TAF have translated into reduced risk of nephrotoxicity and smaller decrease and even increase of BMD in pivotal trials of the TAF-containing single-tablet regimen (STR) of elvitegravir (EVG; E)/cobicistat (COBI; C)/FTC (F)/TAF (E/C/F/TAF) compared to E/C/F/TDF [4-7].

Rilpivirine (RPV)/FTC/TAF STR is a fixed-dose combination (FDC) product of three antiretrovirals, RPV 25mg, FTC $200 \mathrm{mg}$, and TAF $25 \mathrm{mg}$. RPV/FTC/TAF represents the next advancement in non-nucleoside reverse transcriptase inhibitor (NNRTI)-based STRs with expected improvements in renal and bone safety resulting from replacing the TDF component with the new TFV prodrug, TAF, while maintaining the adherence-enhancing convenience of one pill, once daily dosing.

The development of FDCs can be supported by formal bioequivalence evaluations when substantial clinical data for the FDC components exist. The bioequivalence strategy, which compares the pharmacokinetics (PK) of the test coformulation to the PK of the approved antiretroviral components, expedites the approval process since clinical evaluations with the STRs may not be necessary at the time of filing given the ability to extrapolate to often robust and long-term safety and efficacy data. To date, 3 of the 5 available antiretroviral STRs (efavirenz [EFV]/FTC/TDF [Atripla], RPV/FTC/TDF [Complera/ Eviplera], and dolutegravir/lamivudine/abacavir [Triumeq]) have been approved by regulatory authorities for the treatment of HIV-1 infection based on a bioequivalence strategy [8-10]. As such, this study was conducted to establish the bioequivalence (PK comparability) between components of the RPV/FTC/TAF STR and the reference products for each component that are supported by clinical safety and efficacy data (RPV and E/C/F/TAF).

\section{Methods}

\section{Study population}

A total of 96 HIV-negative male and nonpregnant, nonlactating female subjects, between 18 and 45 years of age (inclusive), with a body mass index (BMI) between 19 and $30 \mathrm{~kg} / \mathrm{m}^{2}$ (inclusive), in general good health, and a creatinine clearance $\geq 70 \mathrm{~mL} /$ minute (using the Cockcroft Gault formula and actual body weight) were enrolled in the bioequivalence study.

*Corresponding author: Chuck S, Gilead Sciences Inc, Foster City, CA, United States, Tel: (650)522-3000; (650)522-5801; E-mail: susan.chuck@gilead.com

Received December 21, 2015; Accepted January 03, 2016; Published January 10,2016

Citation: Zack J, Chuck S, Chu H, Graham H, Cao H, et al (2016) Bioequivalence of the Rilpivirine/Emtricitabine/Tenofovir Alafenamide Single-Tablet Regimen. J Bioequiv Availab 8: 049-054. doi:10.4172/jbb.1000266

Copyright: (c) 2016 Zack J, et al. This is an open-access article distributed under the terms of the Creative Commons Attribution License, which permits unrestricted use, distribution, and reproduction in any medium, provided the original author and source are credited. 
A negative serum pregnancy test was required for female subjects of childbearing potential. Screening laboratory evaluations (hematology, chemistry, and urinalysis) had to be within the normal range. Inclusion criteria included having a normal 12-lead electrocardiogram (ECG). Subjects who had a history of recurring syncope, palpitations, or recurring, unexplained dizziness; who had an implanted defibrillator or pacemaker; or who had any serious or active medical or psychiatric illness were excluded. They were also excluded if they took any prescription medications or over-the-counter medications including herbal products within 28 days of commencing study drug dosing; exceptions were vitamins, acetaminophen, ibuprofen, and/or hormonal contraceptive medications. Additionally, subjects treated with systemic steroids, immunosuppressant therapies, or chemotherapeutic agents within 3 months of study screening were excluded. Subjects with current alcohol or substance abuse that could potentially interfere with compliance, as judged by the investigator, were excluded. Subjects were restricted, both before and through discharge, from consuming alcohol-containing products; using nicotine-containing products; and consuming grapefruit juice, grapefruits, and Seville orange juice. Consuming caffeine and other methyl-xanthines-containing products are prohibited only on dosing days.

Informed consent was obtained from each subject before initiation of study procedures. The protocol was reviewed and approved by a central institutional review board (Schulman and Associates, Research Triangle Park, NC). The study was performed in accordance with the principles of the Declaration of Helsinki and adhered to the basic principles of Good Clinical Practice as outlined in the US Code of Federal Regulations (CFR) Title 21, Part 312.

\section{Study design}

This was a randomized, single-dose, open-label, 3-way, 6-sequence, crossover Phase 1 study in healthy adults under fed conditions due to RPV's food requirement (moderate fat meal after at least a 10-hour fasting period). Treatment A contained $25 \mathrm{mg}$ of RPV, $200 \mathrm{mg}$ of FTC, and $25 \mathrm{mg}$ of TAF (RPV/FTC/TAF) STR. Treatment B was a $25 \mathrm{mg}$ tablet of RPV (Edurant). Treatment $\mathrm{C}$ contained $150 \mathrm{mg}$ of EVG, 150 $\mathrm{mg}$ of COBI, $200 \mathrm{mg}$ of FTC, and $10 \mathrm{mg}$ of TAF as a STR (E/C/F/TAF). The duration of the study was either 35 days or 43 days depending on the subject's sequence and included 3 dosing periods. Period 1 and 2 doses were followed by a 14-day washout. Following period 3 dosing, subjects received a follow-up phone call $7( \pm 2)$ days after discharge from the study clinic.

Subjects were randomized to 1 of 6 treatment sequences (ABC [1], ACB [2], BAC [3], BCA [4], CAB [5], and CBA [6]) as described in Table 1 . Subjects on treatment sequences 1 and 3 were discharged on Day 35 while subjects on treatment sequences 2, 4, 5, and 6 ending with RPV-based treatment were discharged on Day 43. A single dose of study drug was administered on Days 1, 15, and 29 within 5 minutes of completing a standardized breakfast (approximately 600 calories and approximately $27 \%$ fat). Following study drug administration, subjects were restricted from food intake until after the 4-hour pharmacokinetic blood sampling time point. For Treatment A (RPV/ FTC/TAF), Treatment B (RPV), and Treatment C (E/C/F/TAF) serial blood samples for PK assessments were collected at the following time points: 0 (predose), $0.25,0.5,0.75,1,1.5,2,3,4,5,6,8,12,24,48$, $72,96,120$, and 144 hours after administration of the treatment. For Treatment A (RPV/FTC/TAF) and Treatment B (RPV), serial blood samples were also collected at the following time points: 168, 192, 216, 240, 264, 288, 312, and 336 hours after administration. Plasma PK sampling occurred over 14 days after dosing for RPV-based treatments to fully characterize the drug components due to RPV's long half-life of $\sim 50$ hours [11].

\section{Bioanalytic methods}

Concentrations of RPV, FTC, and TAF in human plasma samples were determined using fully validated high-performance liquid chromatography-tandem mass spectroscopy (LC/MS/MS) bioanalytical methods. All samples were analyzed within the timeframe supported by frozen stability storage data. Briefly, the methodology for RPV, FTC, and TAF was as follows: $50 \mu \mathrm{L}, 100 \mu \mathrm{L}$, and $50 \mu \mathrm{L}$ of human plasma were spiked with internal standards $\left[{ }^{2} \mathrm{H}_{6}\right]$-rilpivirine; $\left[{ }^{13} \mathrm{C}_{1}\right],\left[{ }^{15} \mathrm{~N}_{2}\right]$-emtricitabine; and $\left[{ }^{2} \mathrm{H}_{7}\right]$-GS-7171 (tenofovir derivative), respectively. The RPV sample was then processed by liquid-liquid extraction with methyl tert-butyl ether. The FTC sample was then processed by protein precipitation with methanol. The TAF sample was then processed by protein precipitation with acetonitrile-formic acid. After this processing, the organic solvent was evaporated and an aliquot of the reconstituted sample extract was injected into the LCMS/MS system.

For each method, the results of within-run (intra-assay) and between-run (inter-assay) precision assessments were reported as the coefficients of variation, each expressed as $\% \mathrm{CV}$, and the results of accuracy assessments were reported as the relative error values expressed as \%RE. For RPV, the calibrated range of the method was 1 to $500 \mathrm{ng} / \mathrm{mL}$; all $\% \mathrm{CV}$ values were $<11 \%$ and $\% \mathrm{RE}$ values were within $\pm 6.6 \%$ of $100 \%$. For FTC, the calibrated range of the method was 5 to $3000 \mathrm{ng} / \mathrm{mL}$; all $\% \mathrm{CV}$ values were $<13.6 \%$ and all $\% \mathrm{RE}$ values were within $\pm 7.3 \%$ of $100 \%$. For TAF, the calibrated range of the method was 1 to $1000 \mathrm{ng} / \mathrm{mL}$. All \%CV values were $<9.6 \%$ and all $\% \mathrm{RE}$ values were within $\pm 7.8 \%$ of $100 \%$.

\section{Safety assessments}

Safety was evaluated throughout the study and included physical examination, vital sign measurement, clinical laboratory tests, evaluation of adverse events (AEs), and review of concomitant medications. Treatment-emergent AEs were defined as any AEs with an onset date on or after the study drug start date and up to 30 days after the permanent discontinuation of study drug. Adverse events were coded using the Medical Dictionary for Regulatory Activities (MedDRA), version 17.1. The severity of AEs and laboratory abnormalities was graded according to the Gilead Sciences, Inc. (GSI) Grading Scale for Severity of Adverse Events and Laboratory Abnormalities (grades 1-4).

\section{Pharmacokinetic analysis}

The PK analysis sets for FTC, RPV, and TAF included all randomized subjects who received at least 1 dose of study drug and had at least 1 plasma concentration data point for each analyte. The sample at the $336^{\text {th }}$ hour for Treatments A and B before Days 15 and 29 served as the time 0 (predose) sample for these days. Samples below the limit of quantitation (BLQ) of bioanalytical assays that were taken before study drug administration were given a value of 0 to prevent overestimation of the initial under the plasma concentration-time curve (AUC). For summary statistics, values that are BLQ at postdose time points were treated as one-half the value of the lower limit of quantitation (LLOQ). For AUC, samples that are BLQ at all other time points were treated as missing data in WinNonlin. Furthermore, subjects with predose concentration values $>5 \%$ of the maximum observed plasma concentration of drug $\left(\mathrm{C}_{\max }\right)$ for any period were excluded from the corresponding PK analysis set. 
Pharmacokinetic parameters were estimated using standard of noncompartmental methods [Phoenix WinNonlin', version 6.4.0; Certara USA, Inc., Princeton, NJ] from the plasma concentration-time data of the three treatments. The primary PK parameters are area under the plasma concentration versus time curve extrapolated to infinity $\left(A U C_{\text {inf }}\right)$, area under the plasma concentration versus time curve from time 0 to the last quantifiable concentration $\left(\mathrm{AUC}_{\text {last }}\right)$, and $\mathrm{C}_{\max }$ of FTC, RPV, and TAF. The following PK parameters were calculated for FTC, RPV, TAF, COBI, and EVG: $\mathrm{AUC}_{\text {inf }}, \mathrm{AUC}_{\mathrm{hst}}, \mathrm{C}_{\mathrm{max}}$, time to maximum concentration $\left(\mathrm{T}_{\max }\right)$, and terminal elimination half-life of the drug in plasma $\left(\mathrm{t}_{1 / 2}\right)$.

\section{Statistical analysis}

The primary endpoints were the $\mathrm{PK}$ parameters $\mathrm{AUC}_{\text {last }}, \mathrm{AUC}_{\text {inf }}$ and $\mathrm{C}_{\text {max }}$ of RPV, FTC, and TAF. Bioequivalence of the test (RPV/FTC/ $\mathrm{TAF}$ ) and reference treatments was concluded if the $90 \%$ confidence interval (CI) of the geometric least-squares mean (GLSM) ratio of the PK parameters for each analyte is within the boundaries of $80 \%$ and $125 \%$.

The primary hypothesis of RPV/FTC/TAF's bioequivalence to E/C/F/TAF STR and RPV (Edurant) tablet was tested using a Two One-Sided Tests (TOST) method with a 5\% significance level for each test. Approximately 78 evaluable subjects or 13 evaluable subjects per sequence were estimated to conduct a TOST with $80 \%$ power for the $90 \%$ CI of the GLSM ratio of test vs. reference treatments with regard to AUC and $\mathrm{C}_{\max }$ to be contained within 0.80 and 1.25 ; this assumed a GLSM ratio of 1.05 . If the true GLSM ratio was 1.0, the TOST with 78 evaluable subjects would have at least $94 \%$ power. A total of 96 subjects or 16 subjects per sequence were enrolled for a $20 \%$ overage. SAS software (SAS Institute, Cary, North Carolina, USA) was used to perform the statistical summaries and analyses.

Subject demographic data, baseline characteristics, plasma concentrations, and PK parameters were summarized by treatment using descriptive statistics. For each analyte (FTC, RPV, TAF), the natural logarithmic transformation of $\mathrm{PK}$ parameters $\left(\mathrm{AUC}_{\text {last }}, \mathrm{AUC}_{\text {inf }}\right.$, and $\mathrm{C}_{\max }$ ) were compared between the test and reference treatments by an analysis of variance (ANOVA) using a mixed-effects model with treatment, period, and sequence as fixed effects and subject within sequence as a random effect. SAS ${ }^{\circ}$ PROC MIXED (SAS Institute, Cary, NC) code was used to calculate the treatment comparisons and corresponding $90 \%$ CIs. The safety analysis set included all randomized subjects who received at least 1 dose of study drug, and safety data were collected starting on the date of the first dose of study drug through 30 days after the last dose of study drug. Safety data (including AEs, laboratory data, and vital signs) were summarized by treatment and the incidence of graded AEs and laboratory abnormalities calculated.

\section{Results}

\section{Subject demographics and disposition}

A total of 96 subjects were randomized and received at least 1 dose of study drug. Of the 96 subjects, most were male (71.9\%) and white $(68.8 \%)$. At baseline, the median age was 32 years (range of 19 to 45 years), median [interquartile range (IQR)] BMI was $26.4 \mathrm{~kg} / \mathrm{m}^{2}$ (23.8-28.3), and median (IQR) creatinine clearance by Cockcroft-Gault method was $120.5 \mathrm{~mL} / \mathrm{min}(106.2-137.2) \mathrm{mL} / \mathrm{min}$. Two subjects did not complete the study: 1 subject did not complete study drug dosing and withdrew consent, and 1 subject completed study drug dosing and withdrew consent.

\section{Pharmacokinetics}

The PK analysis sets for FTC and TAF included all 96 subjects while 95 subjects were included in the RPV PK analysis set. Mean (SD) RPV, FTC and TAF plasma concentration-time profiles are presented in Figure 1 (A-C, respectively). The plasma concentrations of FTC, RPV, and TAF were similar after administration of the test or reference treatment throughout the monitoring period (Treatments $\mathrm{A}$ and B [336 hours], Treatment C [144 hours]). Plasma PK parameters for FTC, RPV, and TAF after administration of the test or reference treatment are presented in Table 2.

FTC and TAF administered as RPV/FTC/TAF (25/200/25 mg) STR met the primary endpoints of the study and demonstrated bioequivalence to E/C/F/TAF (150/150/200/10 mg) STR under fed conditions (moderate fat). RPV administered as RPV/FTC/TAF STR also demonstrated bioequivalence to RPV $25 \mathrm{mg}$ (Edurant) tablet under fed conditions. The $90 \%$ CIs for the GLSM ratios of the primary $\mathrm{PK}$ parameters $\mathrm{AUC}_{\text {ast }}, \mathrm{AUC}_{\text {inf }}$, and $\mathrm{C}_{\max }$ for test versus reference treatments were within the protocol-specified bioequivalence boundary of $80 \%$ to $125 \%$ for FTC, RPV, and TAF. FTC and TAF administered as

(A)

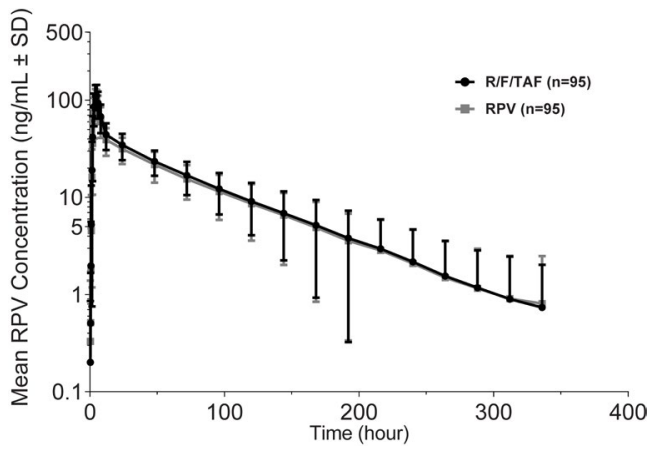

(B)

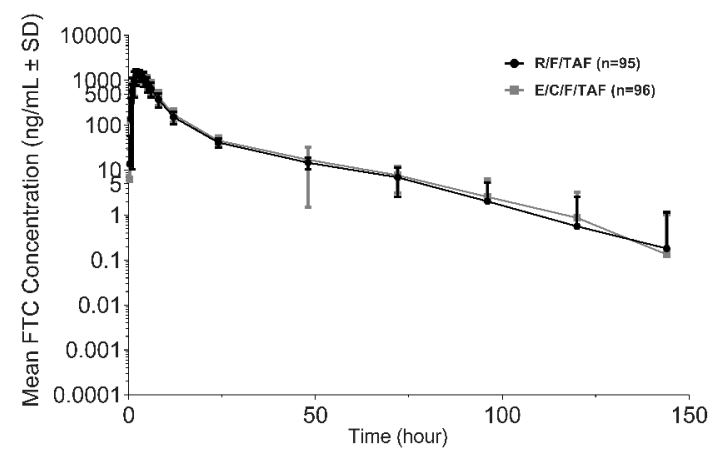

(C)

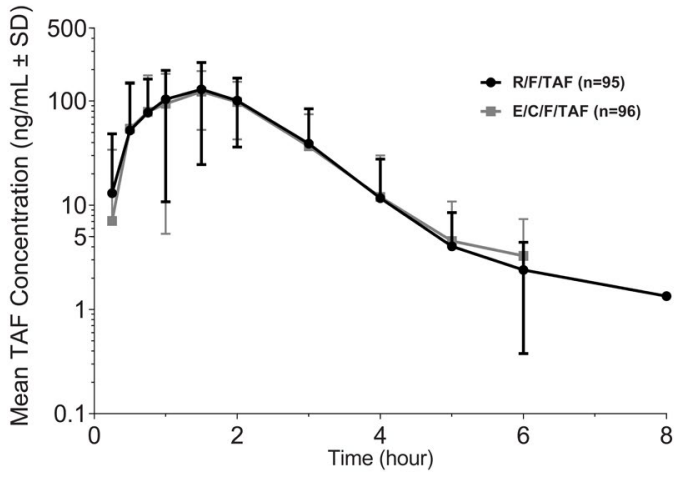

Figure 1A-1C: Mean (SD) RPV, FTC and TAF plasma concentration-time profiles are presented. 


\begin{tabular}{|c|c|c|c|c|c|c|}
\hline $\begin{array}{l}\text { Treatment } \\
\text { Sequence }\end{array}$ & Day 1 & Day 2-14 & Day 15 & Day 16-28 & Day 29 & Discharge \\
\hline 1 & RPV/FTC/TAF & \multirow{6}{*}{ Washout } & RPV & \multirow{6}{*}{ Washout } & $\mathrm{E} / \mathrm{C} / \mathrm{F} / \mathrm{TAF}$ & Day 35 \\
\hline 2 & RPV/FTC/TAF & & $\mathrm{E} / \mathrm{C} / \mathrm{F} / \mathrm{TAF}$ & & RPV & Day 43 \\
\hline 3 & RPV & & RPV/FTC/TAF & & $\mathrm{E} / \mathrm{C} / \mathrm{F} / \mathrm{TAF}$ & Day 35 \\
\hline 4 & RPV & & $\mathrm{E} / \mathrm{C} / \mathrm{F} / \mathrm{TAF}$ & & RPV/FTC/TAF & Day 43 \\
\hline 5 & $\mathrm{E} / \mathrm{C} / \mathrm{F} / \mathrm{TAF}$ & & RPV/FTC/TAF & & RPV & Day 43 \\
\hline 6 & $\mathrm{E} / \mathrm{C} / \mathrm{F} / \mathrm{TAF}$ & & RPV & & RPV/FTC/TAF & Day 43 \\
\hline
\end{tabular}

Table 1: Summary of treatment sequences.

\begin{tabular}{|c|c|c|c|c|c|c|}
\hline \multirow[b]{2}{*}{ PK Parameter ${ }^{\mathrm{a}}$} & \multicolumn{2}{|c|}{ FTC } & \multicolumn{2}{|r|}{ RPV } & \multicolumn{2}{|c|}{ TAF } \\
\hline & $\begin{array}{c}\text { Test: RPV/FTC/TAF } \\
(\mathrm{N}=95)\end{array}$ & $\begin{array}{l}\text { Reference: } E / C / F / T A F \\
(N=96)\end{array}$ & $\begin{array}{l}\text { Test: RPV/FTC/ } \\
\text { TAF (N=95) }\end{array}$ & Reference: RPV (N=95) & $\begin{array}{c}\text { Test: RPV/FTC/TAF } \\
\text { (N=95) }\end{array}$ & $\begin{array}{l}\text { Reference: } E / C / F / T A F \\
(N=96)\end{array}$ \\
\hline$A \cup C_{\text {last }}(\mathrm{ng} \cdot \mathrm{h} / \mathrm{mL})$ & $9381.9(21.7)$ & $10159.4(21.5)$ & $3698.6(34.9)$ & $3373.4(40.0)$ & $250.0(43.4)$ & $238.4(36.5)$ \\
\hline$A \cup C_{\text {inf }}(\mathrm{ng} \cdot \mathrm{h} / \mathrm{mL})$ & $9603.2(21.6)$ & $10387.1(21.5)$ & $3843.1(36.2)$ & $3540.7(43.0)$ & $263.6(42.0)$ & $247.4(36.1)$ \\
\hline$t_{1 / 2}(h)$ & $18.71(15.05,25.27)$ & $18.90(15.89,26.43)$ & $\begin{array}{c}51.65(36.83 \\
66.88)\end{array}$ & $52.51(39.29,66.79)$ & $0.42(0.39,0.49)$ & $0.41(0.37,0.48)$ \\
\hline $\mathrm{C}_{\max }(\mathrm{ng} / \mathrm{mL})$ & $1608.6(26.5)$ & $1583.8(23.8)$ & $121.4(26.1)$ & $108.0(28.7)$ & $198.0(57.7)$ & $191.5(48.2)$ \\
\hline $\mathrm{T}_{\max }(\mathrm{h})$ & $2.00(1.50,3.00)$ & $2.00(2.00,3.00)$ & $4.00(4.00,5.00)$ & $4.00(4.00,5.00)$ & $1.50(1.00,2.00)$ & $1.50(1.00,2.00)$ \\
\hline
\end{tabular}

a. Data are mean $(\% \mathrm{CV})$ except for $\mathrm{T}_{\max }$ and $\mathrm{t}_{1 / 2}$, which are reported as median (first quartile, third quartile)

Table 2: Summary of FTC, RPV, and TAF pharmacokinetic parameters.

\begin{tabular}{|l|c|c|c|c|c|}
\hline & \multicolumn{2}{|c|}{ FTC } & \multicolumn{2}{c|}{ RPV } \\
\hline & $\begin{array}{c}\text { GLSM Ratio (Test/ } \\
\text { Reference) (\%) }\end{array}$ & $\mathbf{9 0 \%}$ Cl (\%) & $\begin{array}{c}\text { GLSM Ratio (Test/ } \\
\text { Reference) (\%) }\end{array}$ & $\begin{array}{c}\mathbf{9 0 \%} \text { Cl (\%) } \\
\text { 90\% CI (\%) } \\
\text { Reference) (\%) }\end{array}$ \\
\hline $\mathrm{AUC}_{\text {last }}(\mathrm{ng} \cdot \mathrm{h} / \mathrm{mL})$ & 92.2 & $90.8,93.7$ & 111.7 & $106.3,117.4$ & 102.9 \\
\hline $\mathrm{AUC}_{\text {inf }}(\mathrm{ng} \cdot \mathrm{h} / \mathrm{mL})$ & 92.4 & $90.9,93.8$ & 110.5 & $105.8,115.4$ & 103.9 \\
\hline $\mathrm{C}_{\max }(\mathrm{ng} / \mathrm{mL})$ & 100.8 & $97.5,104.2$ & 113.5 & $108.4,118.9$ & $98.2,107.8$ \\
\hline
\end{tabular}

Table 3: Statistical comparisons of FTC, RPV, and TAF PK parameters for test vs. reference treatments.

$\mathrm{RPV} / \mathrm{FTC} / \mathrm{TAF}$ and $\mathrm{E} / \mathrm{C} / \mathrm{F} / \mathrm{TAF}$ had similar median $\mathrm{T}_{\text {max }}$ and $\mathrm{t}_{1 / 2}$ values. Rilpivirine administered as RPV/FTC/TAF and RPV (Edurant) tablet also had similar median $\mathrm{T}_{\max }$ and $\mathrm{t}_{1 / 2}$ values. The statistical analyses of FTC, RPV, and TAF PK parameters between test and reference treatments are presented in Table 3.

\section{Safety}

All treatments were generally well tolerated by the study subjects. All AEs were Grade 1 in severity. The most frequently reported AEs were constipation (9.4\%), nausea, and headache (6.3\% each). Treatment-related AEs included nausea and vomiting (4 subjects each) and headache (1 subject) with E/C/F/TAF, and nausea (1 subject) with RPV. No treatment-related AEs were reported with RPV/FTC/TAF. Overall, no deaths or serious AEs were reported, and no AEs led to premature study drug discontinuation.

No laboratory abnormality was considered clinically significant. Five of eight subjects with occult blood on urinalysis had confirmed menses and no events were deemed clinically significant. One subject had Grade 3 amylase and Grade 4 lipase, but did not exhibit clinical symptoms. No clinically relevant changes in median values for hematology and chemistry parameters were observed. No clinically relevant changes in vital sign measurements were observed. No pregnancies were reported during the study.

\section{Discussion}

The results of this study demonstrate the bioequivalence of RPV/ FTC/TAF STR to the reference RPV (Edurant) tablet and E/C/F/TAF STR under fed conditions. The reference of E/C/F/TAF STR was used for the F/TAF components. While this reference contains additional antiretrovirals (EVG and $\mathrm{COBI}$ ), it allows for the extrapolation to the multiple pivotal registrational trials with $\mathrm{E} / \mathrm{C} / \mathrm{F} / \mathrm{TAF}$ that already establish the safety and efficacy of TAF in combination with other ARVs in HIV-1 infected patients. Given this study found the plasma tenofovir alafenamide exposures were bioequivalent for F/TAF 200/25 $\mathrm{mg}$ in the unboosted regimen of RPV/FTC/TAF compared to F/TAF 200/10 mg in boosted EVG and COBI regimen ( 250 vs. $238 \mathrm{ng} \cdot \mathrm{h} / \mathrm{mL}$ ), the RPV/FTC/TAF STR would be expected to have a $>90 \%$ decrease in plasma tenofovir exposure similar to that reported in the pooled Phase 3 treatment-naïve studies of $\mathrm{E} / \mathrm{C} / \mathrm{F} / \mathrm{TAF}$ [4]. This significant reduction in plasma tenofovir exposures is felt to explain the reduced off-target effects of tenofovir on the bone and kidneys observed in the clinical trials of TAF. The reference for the RPV component was RPV (Edurant) tablet to extrapolate to the registrational RPV studies and Phase $3 \mathrm{~b}$ studies of RPV/FTC/TDF STR, which established the safety and efficacy of RPV in HIV-1 infected patients.

In the pooled analysis of two randomized, double-blind, phase 3, non-inferiority studies in treatment-naïve, HIV-infected adults, $\mathrm{E} / \mathrm{C} / \mathrm{F} / \mathrm{TAF}$ not only demonstrated non-inferior efficacy to $\mathrm{E} / \mathrm{C} / \mathrm{F} /$ TDF at Weeks 48 and 96, but also had significantly less proteinuria, no discontinuations due to renal adverse events (AEs), and significantly less decrease in spine and hip BMD [4,12]. Similarly, in two phase 3 studies of virologically suppressed adults who switched off other antiretroviral regimens, E/C/F/TAF maintained high rates of virologic suppression at Week 48 and resulted in significant improvement in proteinuria, no cases of proximal renal tubulopathy or Fanconi syndrome, and increases in spine and hip BMD $[5,6]$. Notably, E/C/F/ TAF also can be safely administered in patients with mild-moderate renal impairment, defined as an estimated glomerular filtration rate (eGFR) of 30-69 mL/min, whereas TDF-containing STRs cannot be administered in patients with eGFR $>50 \mathrm{~mL} / \mathrm{min}$ due to the need to dose adjust TDF in renal impairment [2]. 
The safety and efficacy of the RPV component of this new TAFbased STR has been established in studies of RPV as an individual antiretroviral (Edurant) given with 2 NRTIs and as an STR coformulated with FTC/TDF (Complera/Eviplera). Three randomized, phase 3/3b studies have demonstrated RPV+2 NRTIs and RPV/FTC/TDF STR to have non-inferior efficacy in treatment-naïve, HIV-1-infected adults compared to EFV + 2 NRTIs and EFV/FTC/TDF STR (Atripla) at Weeks 48 and 96 [13-17]. Based on the ECHO and THRIVE data, RPV-based regimens are indicated for treatment-naïve HIV-1 patients with HIV-1 RNA $\leq 100,000$ copies/mL $[11,18]$. RPV/FTC/ TDF STR maintained high rates of virologic suppression through Week 48 in virologically-suppressed patients (HIV-1 RNA $<50$ copies/mL for $\geq 6$ months) switching from ritonavir-boosted PIbased regimens or EFV/FTC/TDF who had no history of virologic failure and were sensitive to all components of the STR $[19,20]$. RPV/FTC/TDF STR was well tolerated with $\leq 2.5 \%$ of treatmentnaïve or virologically suppressed adults discontinuing due to AEs through Week $48[16,19]$. RPV does not require dose adjustment in patients with mild or moderate renal impairment $[11,18]$.

A survey of HIV-infected patients revealed that the top HIV regimen attributes that impact adherence in descending order are: 1) total pills per day, 2) dosing frequency, 3) adverse effects, 4) diet restrictions, 5) pill size, 6-9) monthly number of refills, copayments, prescriptions, medication bottles, and 10) requirement of bedtime dosing [21]. The RPV/FTC/TAF STR effectively addresses 9 of the 10 regimen attributes contributing to adherence, with the exception being the requirement to take it with food. RPV/FTC/TAF STR has the flexibility of being dosed with food any time during the day. The long plasma (RPV 50 hours) and intracellular (FTC $\sim 39$ hours and TFV-DP $\sim 150$ hours) half-lives supported the development of a once daily single-tablet regimen of RPV/ FTC/TAF $[11,22,23]$. STRs provide patients with convenient regimens that improve adherence and eliminate selective non-adherence, and consequently, may lead to improved long-term virological suppression. When approved by Regulatory Authorities, RPV/FTC/TAF will be the smallest commercially available STR measuring approximately 15.4 $\mathrm{mm} \times 7.3 \mathrm{~mm} \times 5.9 \mathrm{~mm}$, which provides an advantage over other STRs for those patients where pill size is a concern.

The third most important attribute impacting adherence in the survey was the occurrence of adverse events. In addition to the differences in adverse events between TDF and TAF, RPV/FTC/TAF may benefit from RPV's tolerability profile compared to EFV [1320,24]. RPV-based STRs have the additional advantages of minimal drug interactions compared to RTV- or COBI-boosted regimens and approximately 5 years of clinical experience with RPV $[1,11]$.

In conclusion, RPV/FTC/TAF STR is bioequivalent to its references, RPV (Edurant) tablet and E/C/F/TAF STR. Importantly, the safety and efficacy data from the registrational trials of RPV and $\mathrm{E} / \mathrm{C} / \mathrm{F} / \mathrm{TAF}$, as well as the phase $3 \mathrm{~b}$ clinical trials with RPV/FTC/TDF, can be extrapolated to RPV/FTC/TAF STR. This bioequivalence study also demonstrated that the RPV/FTC/TAF STR provides therapeutic plasma concentrations of its component antiretroviral agents, which was the basis for initiating 2 on-going Phase 3 safety and efficacy studies of RPV/FTC/TAF to further support its clinical use [clinicaltrials.gov NCT02345226 and NCT02345252]. It is anticipated that these BE results will support the registration of the RPV/FTC/TAF STR to add to the armamentarium of STRs available for efficacious, safe, convenient, and well-tolerated therapies for the treatment of HIV-1 infection.

\section{References}

1. Department for Health and Human Services (DHHS) (2015). Guidelines for the use of antiretroviral agents in HIV-1-infected adults and adolescents. Developed by the DHHS panel on antiretroviral guidelines for adults and adolescents - A working group of the office of AIDS research advisory council (OARAC).

2. Gilead Sciences Inc. VIREAD ${ }^{\circledR}$ (tenofovir disoproxil fumarate) tablets, for oral use. US Prescribing Information. Revised May 2015.

3. Meyer N, Gallant JE, Hsue P, Song X (2015) Comorbidities of Patients with Human Immunodeficiency Virus (HIV) in the USA - a Longitudinal Analysis of Prevalent HIV Patients Over 11 Years [Presentation]. 17-21 San Diego, CA.

4. Sax PE, Wohl D, Yin MT, Post F, DeJesus E, et al. (2015) Tenofovir alafenamide versus tenofovir disoproxil fumarate, coformulated with elvitegravir, cobicistat and emtricitabine, for initial treatment of HIV-1 infection: two randomised, double-blind, phase 3, non-inferiority trials. Lancet 385: 2606-2615.

5. Mills A, Arribas JR, Andrade-Villanueva J, DiPerri G, Van Lunzen J, et al. (2015) Switching from tenofovir disoproxil fumarate to tenofovir alafenamide in antiretroviral regimens for virologically suppressed adults with HIV-1 infection: a randomised, active-controlled, multicentre, open-label, phase 3 , non-inferiority study. Lancet Infectious Diseases 16: 43-52.

6. Pozniak A, Arribas J, Gupta SK, Post FA, Avihingsanon A, et al. (2015) Safety of Tenofovir Alafenamide in Renal Impairment [Poster 795]. Conference on Retroviruses and Opportunistic Infections; February 23-26, 2015; Seattle, WA

7. Kizito H, Gaur A, Prasitsuebsai W, Rakhmanina N, Chokephaibulkit K, et al. (2015) Changes in renal laboratory markers and bone mineral density in treatment-naïve HIV-1-infected adolescents initiating INSTI-based singletablet regimens containing tenofovir alafenamide (TAF) or tenofovir disoproxil fumarate (TDF) [Presentation \#MOAB0104]. International AIDS Society (IAS) Conference on HIV Pathogenesis, Treatment and Prevention; 19-22 July, 2015; Vancouver, Canada.

8. Mathias AA, Hinkle J, Menning M, Hui J, Kaul S, et al. (2007) Bioequivalence of efavirenz/emtricitabine/tenofovir disoproxil fumarate single-tablet regimen. J Acquir Immune Defic Syndr 46: 167-173.

9. Mathias A, Menning M, Wiser L, Wei X, Dave A, et al. (2012) Bioequivalence of the Emtricitabine/Rilpivirine/Tenofovir Disoproxil Fumarate Single Tablet Regimen. J Bioequiv Availab 4: 100-105.

10. Weller S, Chen S, Borland J, Savina P, Wynne B, et al. (2014) Bioequivalence of a dolutegravir, abacavir, and lamivudine fixed-dose combination tablet and the effect of food. J Acquir Immune Defic Syndr 66: 393-398.

11. Janssen-Cilag Ltd. EDURANT ${ }^{\circledR}$ (rilpivirine) tablets for oral use. US Prescribing Information. Revised August 2015.

12. Wohl D, Oka S, Clumeck N, Clarke A, Brinson C, et al. (2015) A randomized, double-blind comparison of tenofovir alafenamide vs tenofovir disoproxil fumarate, each coformulated with elvitegravir, cobicistat, and emtricitabine, for initial HIV-1 treatment: Week 96 results [Poster LBBPD1/1]. 15th European, AIDS Conference (EACS); 21-24 October, Barcelona, Spain.

13. Cohen CJ, Molina JM, Cahn P, Clotet B, Fourie J, et al. (2012) Efficacy and safety of rilpivirine (TMC278) versus efavirenz at 48 weeks in treatment naive HIV-1-infected patients: pooled results from the phase 3 double-blind randomized ECHO and THRIVE trials. J Acquir Immune Defic Syndr 60: 33-42.

14. Cohen CJ, Molina JM, Cassetti I, Chetchotisakd P, Lazzarin A, et al. (2013) Week 96 efficacy and safety of rilpivirine in treatment-naive, HIV-1 patients in two Phase III randomized trials. AIDS 27: 939-950.

15. Behrens G, Rijnders B, Nelson M, Orkin C, Cohen C, et al. (2014) Rilpivirine versus efavirenz with emtricitabine/tenofovir disoproxil fumarate in treatment-naïve HIV-1-infected patients with HIV-1 RNA $\leq 100,000$ copies/mL: Week 96 pooled ECHO/THRIVE subanalysis. AIDS Patient Care and STDs. 28: 168-175.

16. Cohen C, Wohl D, Arribas JR, Henry K, Van Lunzen J, et al. (2014) Week 48 results from a randomized clinical trial of rilpivirine/emtricitabine/tenofovir disoproxil fumarate vs. efavirenz/emtricitabine/tenofovir disoproxil fumarate in treatment-naive HIV-1-infected adults. AIDS. 28: 989-997.

17. Van Lunzen J, Antinori A, Cohen CJ, Arribas JR, Wohl DA, et al. (2016) Rilpivirine vs. efavirenz-based single-tablet regimens in treatment-naïve adults: Week 96 efficacy and safety from a randomized phase 3b study. AIDS 30: 251-259. 
Citation: Zack J, Chuck S, Chu H, Graham H, Cao H, et al (2016) Bioequivalence of the Rilpivirine/Emtricitabine/Tenofovir Alafenamide Single-Tablet Regimen. J Bioequiv Availab 8: 049-054. doi:10.4172/jbb.1000266

18. Gilead Sciences Inc. COMPLERA ${ }^{\circledast}$ (emtricitabine, rilpivirine, tenofovir disoproxil fumarate) tablets. US Prescribing Information. Revised May 2015.

19. Palella FJ Jr, Fisher M, Tebas P, Gazzard B, Ruane P, et al. (2014) Simplification to rilpivirine/emtricitabine/tenofovir disoproxil fumarate from ritonavir-boosted protease inhibitor antiretroviral therapy in a randomized trial of HIV-1 RNAsuppressed participants. AIDS 28: 335-344.

20. Mills AM, Cohen C, DeJesus E, Brinson C, Williams S, et al. (2013) Efficacy and safety 48 weeks after switching from efavirenz to rilpivirine using emtricitabine/ tenofovir disoproxil fumarate-based single-tablet regimens. HIV Clin Trials 14: 216-233.

21. Stone VE, Jordan J, Tolson J, Miller R, Pilon T (2004) Perspectives on adherence and simplicity for HIV-infected patients on antiretroviral therapy:
Self-report of the relative importance of multiple attributes of highly active antiretroviral therapy (HAART) regimens in predicting adherence. $J$ Acquir Immune Defic Syndr 36: 808-816.

22. Hawkins T, Veikley W, St. Claire RL3rd, Guyer B, Clark N, et al. (2005) Intracellular pharmacokinetics of tenofovir diphosphate, carbovir triphosphate and lamivudine triphosphate in patients receiving triple-nucleoside regimens. $J$ Acquir Immune Defic Syndr 39: 406-411.

23. Molina JM, Cox SL (2005) Emtricitabine: a novel nucleoside reverse transcriptase inhibitor. Drugs Today (Barc) 41: 241-252.

24. Bristol-Myers Squibb and Gilead Sciences Limited. ATRIPLA ${ }^{\circledR}$ (efavirenz emtricitabine, tenofovir disoproxil fumarate) tablets, for oral use. US Prescribing Information. Revised January 2015. 